\title{
A rare case of broad ligament pregnancy diagnosed at 36 weeks of gestation incidentally during laparotomy
}

\author{
Satyajit Jena, Sushree Jagadeb, Jagannath Mishra*, Subhashree Rout
}

Department of Obstetrics \& Gynaecology, SCB Medical College, Cuttack, Odisha, India

Received: 14 March 2015

Revised: 18 March 2015

Accepted: 19 April 2015

\section{*Correspondence:}

Dr. Jagannath Mishra,

E-mail: jagannathmishra05@gmail.com

Copyright: ( $)$ the author(s), publisher and licensee Medip Academy. This is an open-access article distributed under the terms of the Creative Commons Attribution Non-Commercial License, which permits unrestricted non-commercial use, distribution, and reproduction in any medium, provided the original work is properly cited.

\begin{abstract}
Pregnancy in the broad ligament is a rare form of ectopic abdominal pregnancy with a high risk of maternal mortality. Ultrasound examination may help in the early diagnosis but mostly the diagnosis is established during surgery. We are reporting a case of broad ligament pregnancy diagnosed incidentally during laparotomy. She had uneventful postoperative recovery.
\end{abstract}

Keywords: Broad ligament, Laparotomy

\section{INTRODUCTION}

Abdominal pregnancies account for $1 \%$ of ectopic pregnancies and the maternal mortality rate has been reported to be as high as $20 \% .^{1,2}$ It may occur in any part of the abdomen but is common in pouch of Douglas and is rare in broad ligament. It presents as an acute abdominal emergency during pregnancy and the diagnosis is commonly achieved during surgical exploration. Though ultrasonography may at times suggest this possibility if there is high index of suspicion.

\section{CASE REPORT}

A gravida-2 para-1 at 36 weeks came to our labor room after being referred from a rural hospital with complains of bleeding per vaginum and pain abdomen for last 3 days. She had an ultrasonography report showing single dead fetus of 35 weeks with vertex presentation with fundal placenta. Estimated fetal weight was $2.5 \mathrm{~kg}$. On examining the patient she was looking pale. Per abdominal examination revealed uterus term size, relaxed with cephalic presentation. On auscultation fetal heart sound was not localized. On per speculum examination cervix was deviated to right side. On bimanual examination cervix was deviated to right side and os was closed. Head was felt posterior to the os towards the left side. Induction of labor was started with tablet misoprostol (50 micrograms repeated 4hourly for four doses). As there was no progress after repeated doses of misoprostol and Foley's catheter couldn't be negotiated through cervix, she was planned for termination by caesarean section.

After opening the peritoneal cavity we found uterus of size 18-20wks and it was deviated to right side. After opening the uterus no product of conception was found inside the cavity (Figure 1). On examining the left side broad ligament fetal parts were palpable. Therefore suspecting a broad ligament pregnancy, the broad ligament cavity was opened by cutting on anterior layer of broad ligament. A macerated female baby (Figure 2) was delivered followed by placenta and membranes. The placenta was implanted over the broad ligament (Figure $3,4)$. Uterus was explored and it was found to be intact without any rupture (Figure 5). Feeding vessels were seen 
on the back from omentum and rectum (Figure 6). Right side tube and ovary was healthy but left side adnexal anatomy was grossly distorted. Uterus was closed followed by closure of the anterior layer of broad ligament. A corrugated rubber drain was given in the broad ligament cavity as there was oozing from the placental bed. Abdomen was closed.

The patient recovered uneventfully and drain was removed after 48 hours and was discharged on $8^{\text {th }}$ postoperative day.

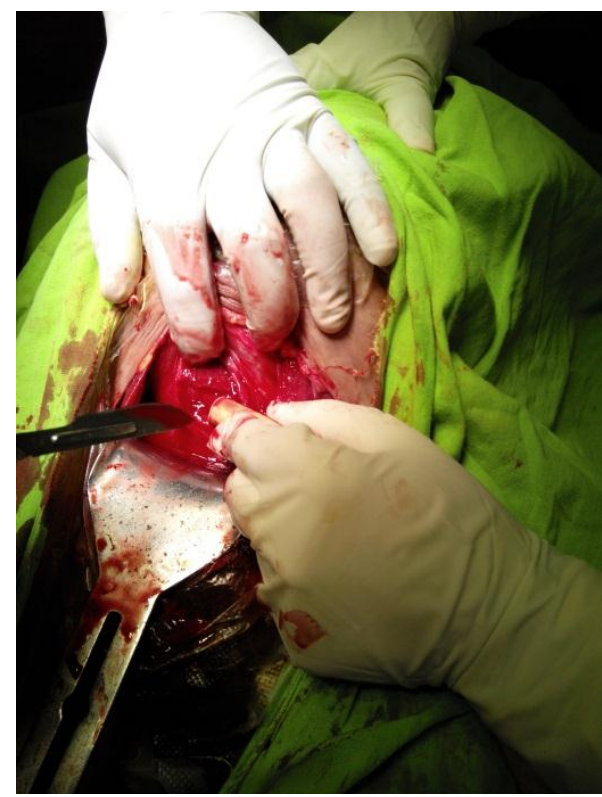

Figure 1: Showing the cavity is empty after opening the anterior wall of uterus.

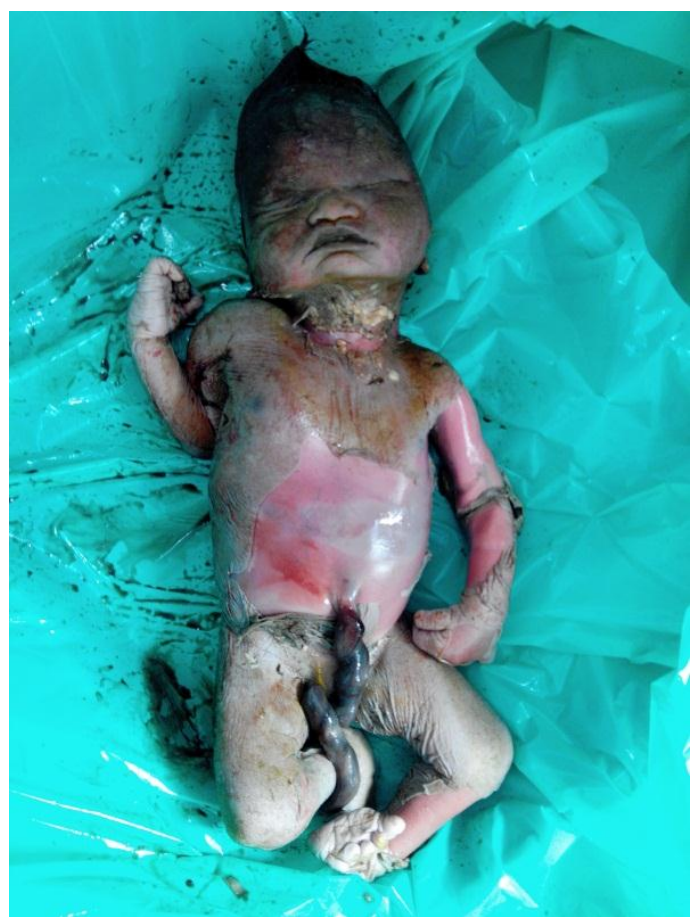

Figure 2: The macerated fetus.

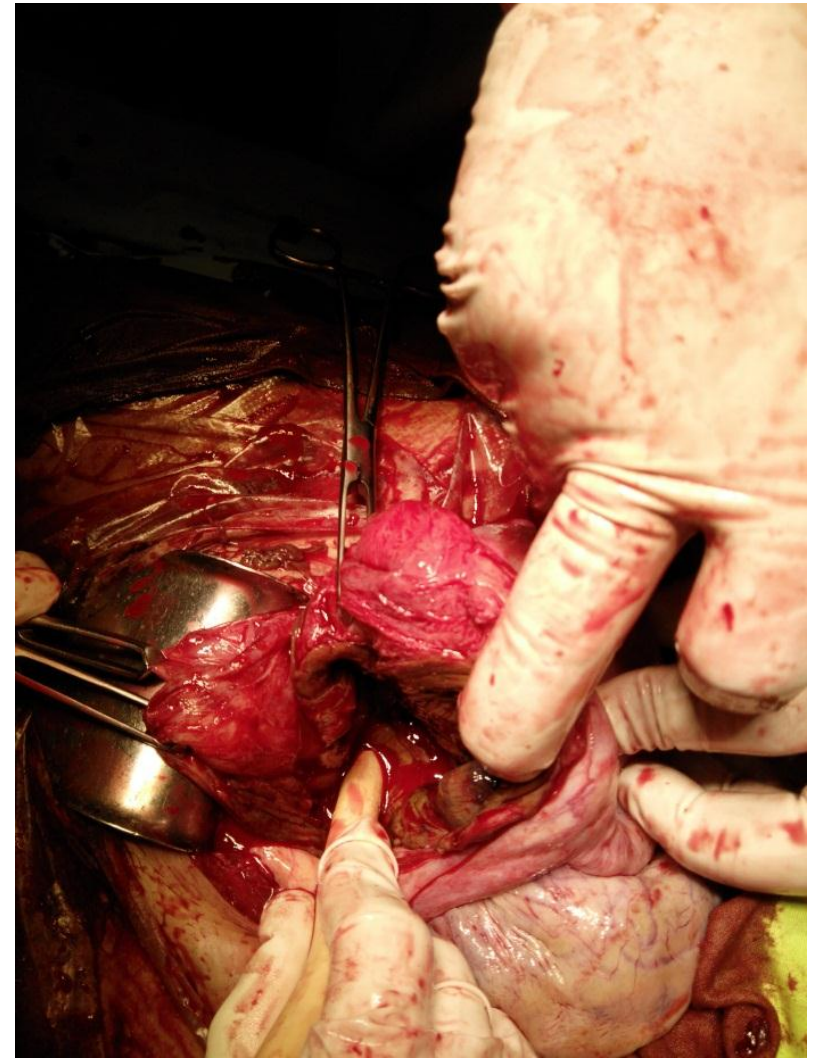

Figure 3: Showing the site of implantation of placenta over the newly formed broad ligament cavity.

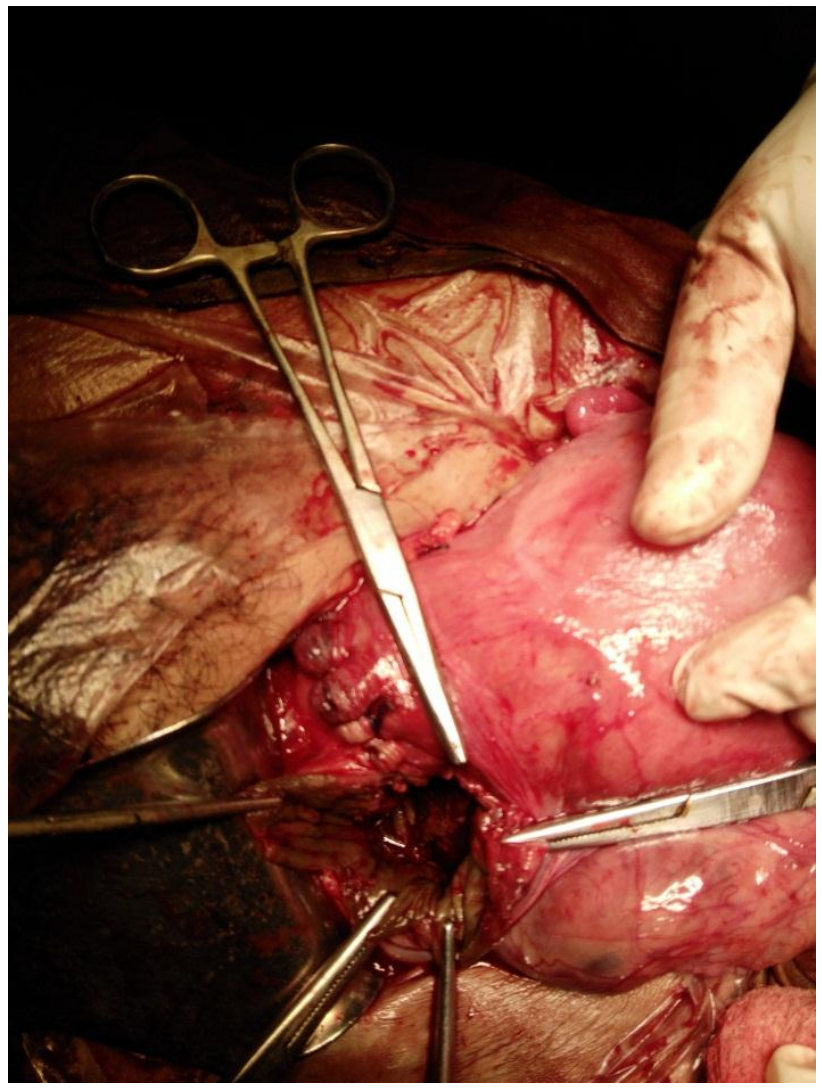

Figure 4: The implantation of placenta clearly over the broad ligament after closing the cut uterine wall. 


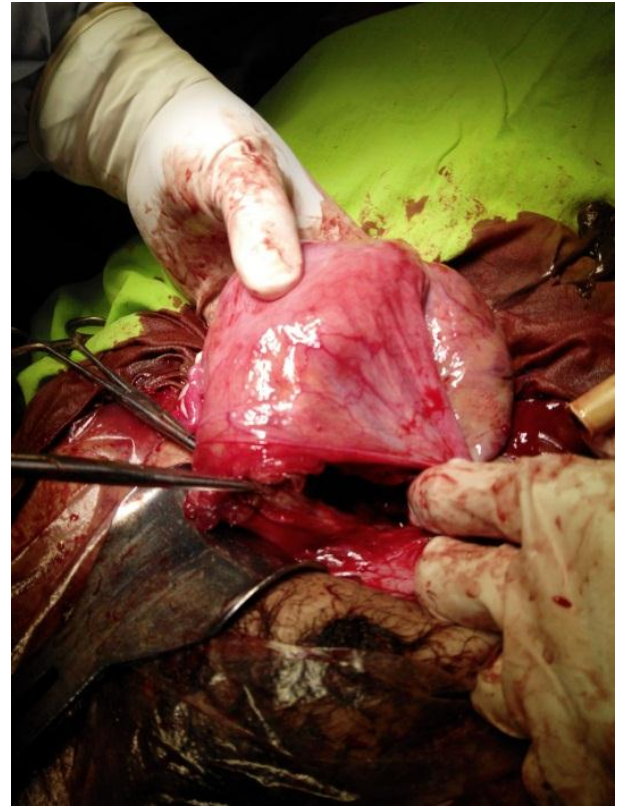

Figure 5: When explored the uterine wall it was found to be intact.

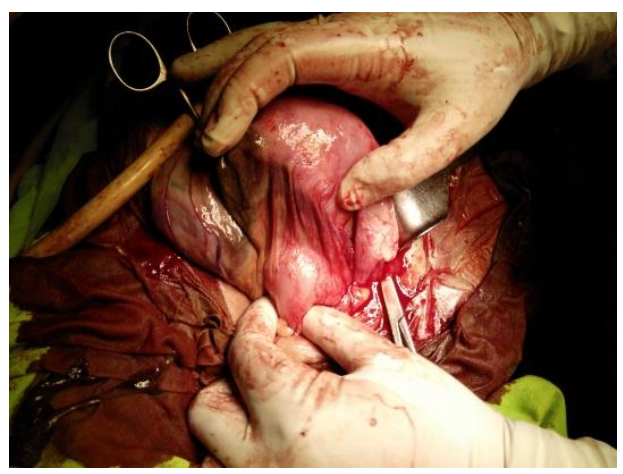

Figure 6: Showing the posterior surface of broad ligament cavity, rectum is clearly visible.

\section{DISCUSSION}

Broad ligament ectopic pregnancy is a rare but life threatening condition. The abdominal pregnancies account for $1 \%$ of ectopic pregnancies. Maternal mortality is as high as $20 \%$. It is either due to primary implantation of the zygote on the broad ligament or followed by secondary implantation to fallopian tube, ovary or other peritoneal surface. The risk factors include a history of secondary infertility, pelvic inflammatory disease, use of intrauterine devices, use of progesterone only pills, a previous history of ectopic pregnancy and endometriosis. ${ }^{3}$ There are various clinical presentations reported in the literature but a dull lower abdominal pain during early gestation is common. This has been attributed to the placental separation, tearing of broad ligament and small peritoneal haemorrhage., ${ }^{4,5}$ Vaginal bleeding is also a common feature reported in up-to half of the patients as reported by Hallalt and Grove. ${ }^{6}$ This bleeding is reported to be due to breakdown of decidual casts. ${ }^{3,4}$ It has been described in the literature that if there is no intrauterine pregnancy on ultrasonography and the ectopic sac is beside the lower part of uterus, a strong suspicion of broad ligament ectopic should be considered. MRI provides additional information and may help in surgical planning by evaluating the extent of uterine and mesenteric involvement. ${ }^{7,8}$ The management is exploratory laparotomy. However, stable patients with early gestation can be considered for laparoscopic removal for small broad ligament pregnancies.9 Conservative management or medical management is not recommended for broad ligament ectopic if the diagnosis is certain.

Funding: No funding sources

Conflict of interest: None declared

Ethical approval: Not required

\section{REFERENCES}

1. Ludwig M, Kaisi M, Bauer O, Diedrich K. The forgotten child - a case of heterotropic, intraabdominal and intrauterine pregnancy carried to term. Hum Reprod. 1999;14:1372-4.

2. Alto WA. Abdominal pregnancy. Am Fam Physician. 1990;41:209-14.

3. Cordero DR, Adra A, Yasin S, O'Sullivan MJ. Intraligamentary pregnancy. Obstet Gynecol Surv. 1994;49:206.

4. Paterson WG, Grant KA. Advanced intraligamentous pregnancy. Report of a case, review of the literature and a discussion of the biological implications. Obstet Gynecol Surv. 1975;30:715-26.

5. Vierhout ME, Wallenburg HC. Intraligamentary pregnancy resulting in a live infant. Am J Obstet Gynecol. 1985;152:878-9.

6. Hallatt JG, Grove JA. Abdominal pregnancy: a study of 21 consecutive cases. Am J Obstet Gynaecol. 1985;152:444-9.

7. Malian V, Lee JH. MR imaging and MR angiography of abdominal pregnancy with placental infarction. Am J Roentgenol. 2001;177:1305-6.

8. Yoshigi J, Yashiro N, Kinoshito T, O'uchi T, Kitagaki H. Diagnosis of ectopic pregnancy with MRI: efficacy of $\mathrm{t} 2$ weighted imaging. Magn Reson Med Sci. 2006;5:25-32.

9. Pisarka MD, Casson PR, Moise KJ Jr, Di Maio DJ, Buster JE, Carson SA. Heterotropic abdominal pregnancy treated at laparoscopy. Fertil Steril. 1998;70:159-60.

DOI: 10.18203/2320-1770.ijrcog20150116

Cite this article as: Jena S, Jagadeb S, Mishra J, Rout S. A rare case of broad ligament pregnancy diagnosed at 36 weeks of gestation incidentally during laparotomy. Int J Reprod Contracept Obstet Gynecol 2015;4:875-7. 